Comparative Population Studies

Selected Articles in German Vol. 40 (2015): 33-52

(Erstveröffentlichung: 25.02.2015)

\title{
Der Eintritt in die nachelterliche Familienphase
}

\section{Barbara Wawrzyniak}

Zusammenfassung: Der Beitrag befasst sich mit dem Eintritt in die nachelterliche Familienphase, also die familiäre Situation, bei der alle Kinder aus dem elterlichen Haushalt ausgezogen sind. Zum einen wird dieser Eintritt zeitlich im Lebensverlauf positioniert und zum anderen das Übergangsrisiko untersucht. Mit Hilfe von Paneldaten ( 3 Erhebungswellen) über einen Zeitraum von 40 Jahren einer Kohorte ehemaliger nordrhein-westfälischer Gymnasiasten wird mittels eines ereignisanaIytischen Verfahrens (Cox-Regression) analysiert, welche Faktoren den Übergang beschleunigen bzw. verlangsamen. Es zeigt sich, dass die private Lebensbiografie (insbesondere das Alter beim eigenen Auszug, das Alter bei der Geburt des ersten Kindes und die Kinderzahl) der Eltern einen hohen Einfluss auf den Eintrittszeitpunkt besitzt, während der berufliche Werdegang keinen Effekt hat. Darüber hinaus verzögern Söhne den Übergang, während ein Studium und eine Berufstätigkeit der Kinder diesen beschleunigen.

Schlagwörter: Nachelterliche Familienphase · Auszug letztes Kind · Empty nest · Panelstudie $\cdot$ Cox-Regression

\section{Die nachelterliche Familienphase}

Wenn Kinder erwachsen werden und das elterliche Haus verlassen, dann beginnt für die Eltern ein neuer Abschnitt im Leben: die nachelterliche Phase. Dieser Lebensabschnitt entwickelte sich erst im 20. Jahrhundert zu einer über Jahre andauernden Phase, die 25 bis $35 \%$ der gesamten Lebensspanne einnimmt (Lauterbach 2004: 229). Durch die Verlängerung dieses Lebensabschnitts müssen die Beziehungen zwischen erwachsenen Kindern und ihren Eltern länger gestaltet werden als zwischen minderjährigen Kindern und ihren Eltern, denn die Zeit der Pflege und Versorgung der Kinder beträgt nur noch ein Viertel der gesamten Lebenszeit (NaveHerz 2002: 57). Der Eintritt in die nachelterliche Phase ist weniger institutionell gerahmt als eine Hochzeit oder die Geburt eines Kindes (Scherger 2007). 
Für die Eltern birgt dieser Lebensabschnitt neue Herausforderungen für die Konsolidierung der Beziehungen zwischen den Partnern und den Kindern. Es steht nicht mehr die Betreuung der Nachkommen im Vordergrund, der familiäre Alltag verändert sich. Frühere Studien berichten gar vom empty-nest-Syndrom, das mit Depressionen und anderen psychischen Erkrankungen der Mütter einhergeht (z.B. Bart 1971; Deykin et al. 1966). In späteren Forschungen hingegen wird das Aufkommen dieser Belastungen widerlegt (z.B. Radloff 1980) und eher auf ambivalente Gefühle der Mütter verwiesen (Feser et al. 1989). Papastefanou (2000) berichtet sogar von positiven Bewertungen der räumlichen Trennung durch die Familienmitglieder. Der räumliche Abstand bewirkt eine Reduzierung der alltäglichen Reibereien zwischen Eltern und Kindern und die Erfahrung des Alleinlebens mit all seinen Herausforderungen führt dazu, dass die jungen Erwachsenen ihren Eltern mehr Verständnis entgegenbringen (Papastefanou 2000: 62).

Der Auszug der eigenen Kinder kann sowohl positiv als auch negativ gewertet werden, je nachdem welches Verhältnis zu den Kindern besteht und ob der Auszug als Schritt zur gewünschten Selbstständigkeit gesehen wird oder aber als frühzeitige "Flucht" aus dem Elternhaus. Darüber hinaus tangiert der Auszug sowohl den Lebensbereich Elternschaft als auch den Bereich Partnerschaft: „... einerseits wird als schmerzlich empfunden, daß [sic] der alltägliche Kontakt mit den Kindern wegfällt; andererseits wird klar gesehen, daß [sic] die individuelle und partnerschaftliche Entwicklung profitiert" (Papastefanou 2000: 58). Allerdings beeinträchtigt eine starke Fixierung auf die Elternrolle die Verarbeitung des Übergangs in die nachelterliche Phase (Papastefanou 2000: 58). Vor allem für die Mütter, die ihre Erwerbstätigkeit für die Familie aufgegeben haben, bildet der Auszug ihrer Kinder eine schwierige Situation: „Da Mütter eine ihrer wichtigsten identitätsstiftenden Aufgaben verlieren, entsteht oft das Gefühl, nicht mehr gebraucht zu werden, verbunden mit Depressivität, Trauerreaktion und einer Identitätskrise." (Pinquart/Silbereisen 2007: 498) Doch gibt es auch Studien, die diese Phase ebenfalls mit positiven Erfahrungen für die Frauen belegen, so konnte eine Abnahme negativer und eine Zunahme positiver Gefühle bei Müttern, deren Kinder ausgezogen sind, ausgemacht werden. Auch ein Anstieg des allgemeinen Wohlbefindens und die Reduktion kleiner Alltagsärgernisse wurden festgestellt (Dennerstein et al. 2002). Für Väter hingegen stellt sich heraus, dass diese wenige Probleme mit dem Auszug der Kinder haben. Allerdings kann sich bei sehr hohem beruflichem Engagement ein Gefühl des Bedauerns einstellen, dass sie das Aufwachsen der Kinder versäumt haben. Und bei Vätern, die sich in unglücklichen Beziehungen befinden, in denen die Kinder gelegentlich als Integrationsfaktor wirkten, können ebenso Schwierigkeiten auftreten (Papastefanou 1997).

Die Beziehungsqualität zwischen den erwachsenen Kindern und ihren Eltern zeichnet sich in dieser Phase durch eine geringe räumliche Distanz und eine große Enge aus, wobei zwischen Müttern und Töchtern die größte Nähe besteht. Von einem Auseinanderleben der Generationen kann nicht die Rede sein (Szydlik/Schupp 1998: 311). Allerdings bewerten Eltern ihre Beziehung zu den eigenen Kindern enger als die Kinder sie wahrnehmen, sie neigen auch eher dazu, die intergenerationalen Gemeinsamkeiten hervorzuheben und Konflikte herunterzuspielen, während die 
Kinder die Gemeinsamkeiten unter- und die Konflikte überbetonen (Szydlik/Schupp 1998: 304).

Im Zentrum dieses Beitrags steht der Übergang in die nachelterliche Phase, die durch den Auszug des letzten Kindes markiert wird. Zum einen wird geklärt, wie sich der Übergang zeitlich im Lebensverlauf der Eltern positionieren lässt und zum anderen, wovon der Eintritt in die nachelterliche Familienphase abhängt. Dabei wird sowohl der private als auch der berufliche Lebenslauf der Eltern und Kinder in die multivariaten Analysen einbezogen. Zunächst wird der Forschungsstand zum Übergang in die nachelterliche Phase und zum Auszugsverhalten junger Erwachsener dargestellt. Anhand der bisherigen Ergebnisse sollen mögliche Einflussfaktoren ausgemacht und in die Analysen übernommen werden. Anschließend werden die hier verwendete Stichprobe, Methode und Datenbasis erläutert, bevor die deskriptiven und multivariaten Ergebnisse vorgestellt werden.

\section{Determinanten des Einflusses auf den Eintritt in die nachelterliche Phase}

Die Perspektive und Situation der Eltern beim Auszug der eigenen Kinder bzw. des letzten Kindes wurde bislang nur in wenigen Studien beleuchtet (Lauterbach 2004; Mayer/Wagner 1989), weshalb dieser Aspekt nun stärker in den Fokus gerückt wird. Das Auszugsverhalten junger Erwachsener hingegen wurde bereits in mehreren Studien ausgiebig untersucht. Einen Überblick über den Forschungsstand zum Auszugsverhalten und dessen bestimmenden Faktoren findet sich bei Berger (2009). Es lässt sich festhalten, dass sich der Auszugszeitpunkt bei Männern und Frauen unterscheidet (Berger 2009; Ziegler/Schaldt 1993; Mayer/Wagner 1989; Scherger 2009). Frauen verlassen das elterliche Haus früher als Männer, je nach Studie im Schnitt um zwei bis fünf Jahre und meistens am Anfang ihrer Zwanziger. So findet sich bei Berger (2009) ein Altersunterschied zwischen 21,2 Jahren bei Frauen und 22,8 Jahren bei Männern (Median), während Ziegler und Schladt (1993) auf einen geringeren Unterschied kommen und ein früheres Auszugsalter feststellen: 20,6 Jahre bei Frauen und 21,9 Jahre bei Männern (Median).

Darüber hinaus gibt es deutliche Zusammenhänge zwischen dem beruflichen und privaten Lebenslauf und dem Auszugsverhalten junger Erwachsener (Zieg/er) Schladt 1993; Mayer/Wagner 1989). Wobei das Auszugsverhalten in der Bundesrepublik in den letzten Jahrzehnten einem Strukturwandel unterlag: Während früher ein Auszug stark an die Eheschließung gekoppelt war (Mayer/Wagner 1989), ist dieser heutzutage eher mit neuen Lebensformen, wie der nichtehelichen Kohabitation verbunden (Berger 2009). Darüber hinaus erweisen sich die berufliche Ausbildung und der Eintritt in den Arbeitsmarkt als zeitlich enger mit dem Auszug verbunden als früher (Berger 2009: 198).

Ziegler und Schladt (1993) kommen bei der Analyse des Auszugsverhaltens der Kohorte des Kölner Gymnasiastenpanels zu dem Ergebnis, dass der Loslösungsprozess vom Elternhaus nicht von der sozialen Herkunft abhängt, sondern vielmehr von 
Ereignissen und Einschnitten im institutionell geregelten Lebenslauf, ${ }^{1}$ also Übertritte im Bildungssystem, Eintritte ins Erwerbsleben und eine (eheliche oder nichteheliche) Partnerschaft. So konnte auch Berger (2009) aufzeigen, dass eine Erwerbstätigkeit und die damit erreichte ökonomische Selbstständigkeit das Auszugtempo von Frauen und Männern sehr deutlich beschleunigt. Ebenso bewirkt die Aufnahme eines Studiums bei beiden Geschlechtern eine höhere Auszugswahrscheinlichkeit. Die Einflüsse einer Berufsausbildung sind hingegen nicht sehr stark, sie erhöht die Auszugsneigung nicht signifikant. Das Bildungsniveau spielt insofern eine Rolle, als ein längerer Verbleib im Schulsystem zu einem späteren Auszug führt und somit die ökonomische Selbstständigkeit hinauszögert. Die Partnerbeziehung zeigt sich ebenfalls als ein wichtiger Faktor bei der Bestimmung der Auszugswahrscheinlichkeit. Diese steigt deutlich an, wenn junge Erwachsene sich in einer festen, nichtehelichen Beziehung befinden (Berger 2009: 232).

Bei der Entscheidung aus dem Elternhaus auszuziehen spielt ebenso der Wohnkomfort eine wichtige Rolle: Ist dieser hoch und verschlechtert er sich womöglich deutlich nach dem Auszug, so wirkt die Aussicht auf eine Verschlechterung der eigenen Wohnsituation eher hemmend auf die Auszugsentscheidung (Wagner 1989: 173). Die finanziellen Ressourcen der Eltern können sich in zwei Richtungen auf die Auszugsentscheidung auswirken: Zum einen kann ein finanziell gut ausgestatteter Haushalt dazu führen, dass der Wohnkomfort der Kinder hoch ist und somit den Auszug hinauszögert und zum anderen kann ein hohes Haushaltseinkommen der Eltern die eigene Haushaltsführung des Kindes stärker unterstützen und somit zu einem schnelleren Auszug führen (Wagner 1989: 174). Die familiäre Konstellation, gemessen an der Kinderanzahl, kann sich ebenfalls als Faktor auf den Zeitpunkt des Auszugs auswirken: Bei einer hohen Anzahl an Kindern würde man vermuten, dass die älteren Geschwister mit dem Auszug das Bedürfnis nach Privatheit befriedigen können, wohingegen Einzelkinder länger zu Hause verweilen (Lauterbach 2004: 163). Einen weiteren wichtigen Faktor, der allerdings in den folgenden Analysen nicht berücksichtigt wird, stellt die Familienstruktur dar: Stiefkinder und Kinder von Alleinerziehenden ziehen in der Regel früher aus als Kinder, die mit beiden biologischen Eltern zusammenwohnen (Mitche/l 1994). Da in den Daten bereits eine Einschränkung bzgl. des Familienverlaufs vorgenommen wurde, wird der Einfluss von Stieffamilien und Alleinerziehenden ausgeschlossen.

Die genannten Einflüsse auf den Auszug der jungen Erwachsenen sind wichtige Faktoren zur Bestimmung des Eintritts in die nachelterliche Familienphase, jedoch gilt es auch diejenigen zu bestimmen, die das Leben der Eltern kennzeichnen. Ein früher Beginn der nachelterlichen Phase kann das Ergebnis verschiedener Einflüsse sein: frühes Heiratsalter, frühes Erstgeburtsalter, geringe Kinderanzahl und geringer Abstand der Geburten (Mayer/Wagner 1989: 29). Wie sieht es aber mit der Bildung der Eltern aus? Welchen Einfluss besitzt sie auf die zeitliche Positionierung des Eintritts in die nachelterliche Phase aus? Eltern, die über einen Hochschulabschluss

1 Es sind Ereignisse, die durch Institutionen geregelt werden oder gar selber als Institution angesehen werden (z.B. Ehe). 
verfügen, wünschen sich womöglich für ihre Kinder eine adäquate Bildung und können ihnen diese auch finanziell ermöglichen (Meulemann 1995). Dabei kann der Studienabschluss der Eltern den Auszugszeitpunkt der Kinder in zwei Richtungen beeinflussen: Wenn die Kinder in Ortsnähe studieren, wird der Auszug hinausgezögert und wenn sie außerhalb studieren beschleunigt. Scherger (2007) kommt bei ihrem Vergleich zwischen mehreren Kohorten zu dem Ergebnis, dass bei Männern und Frauen mit Abitur die nachelterliche Phase einige Jahre später beginnt als bei denjenigen ohne Abitur, was darauf hinweist, dass Kinder höher gebildeter Eltern längere Bildungsprozesse durchlaufen, die den Auszug verzögern (Scherger 2007: 186). Wagner (1989) stellt in diesem Zusammenhang fest, dass das Bildungsniveau der Eltern eine gegenläufige Wirkung aufweist, wenn der Haushaltstyp der Jugendlichen nach dem Auszug ebenfalls betrachtet wird: Wenn die Kinder bei Auszug heiraten, dann wirkt ein höheres Bildungsniveau der Eltern hemmend auf den Auszug. Wenn sie nicht heiraten, dann beschleunigt das Bildungsniveau den Auszug (Wagner 1989: 182).

Die Berufstätigkeit der Mütter beschleunigt das Auszugsverhalten der Kinder, dabei kann eine berufstätige Mutter zum einen als Rollenvorbild einer größeren Selbstständigkeit - vor allem für die Töchter - dienen und zum anderen durch die weniger ausgeprägte Überbetreuung der Kinder zu einer schnelleren Loslösung führen (Ziegler/Schladt 1993).

In diesem Beitrag sollen, aufbauend auf den bisherigen Forschungsergebnissen, die zentralen Faktoren zum Eintritt in die nachelterliche Phase ausgemacht werden. Dabei wird der Fokus nicht, wie bisher, nur auf einzelne Lebensbereiche der Eltern gelegt, sondern sowohl der Einfluss der Privatbiografie als auch der Berufsbiografie im Längsschnitt betrachtet und ebenso die Merkmale der Kinder einbezogen.

\section{Stichprobe, Methode und Daten}

\subsection{Stichprobe}

Die Analysen werden mit den Daten des Kölner Gymnasiastenpanels (KGP) durchgeführt, bei dem es sich um Daten einer Kohorte der Jahrgänge 1953 bis 1956 handelt. Die Befragten wurden zum ersten Mal im Jahr 1969 schriftlich befragt und waren zu dem Zeitpunkt Schüler der 10. Klasse an nordrhein-westfälischen Gymnasien. Von den damals 3240 Teilnehmern konnten im Jahr 1984/85 1987 Personen für eine persönliche Wiederbefragung gewonnen werden. Eine zweite Wiederbefragung wurde im Jahr 1997 mit 1596 Personen telefonisch durchgeführt und schließlich eine dritte Erhebungswelle im Jahr 2010 mit 1301 Teilnehmern, die ebenfalls telefonisch durchgeführt wurde. Der Fragebogen umfasst einen biografischen Teil, der die Schul-, Ausbildungs-, Studiums-, Erwerbs-, Familien- und Partnerbiografie abdeckt. Darüber hinaus gibt es eine Reihe von Einstellungs- und Freizeitfragen sowie in der aktuellen Erhebung Fragen zur Vorbereitung auf das Alter. Je nach Erhebungswelle wurde ein anderer Themenschwerpunkt gewählt, so wurde bei der ersten Wiederbefragung die Identitätsbildung des Jugendlichen zwischen dem 16 . und 
30. Lebensjahr in den Vordergrund gerückt, während bei der zweiten Wiederbefragung die Identitätswahrung des Erwachsenen zwischen dem 30. und 43. Lebensjahr beleuchtet wurde. Die dritte Welle befasst sich mit der Weiterentwicklung der Identität und der Vorbereitung auf das Alter. Besonders vorteilhaft ist, dass für die 1301 Befragten, die 2010 an der dritten Erhebungswelle teilgenommen haben, die gesamte Partnerschafts- und Familienbiografie vom 16. bis zum 56. Lebensjahr aufgezeichnet ist. Somit lässt sich für diese Kohorte eine genaue Übersicht über Dauer und Verlauf der Partnerschaften sowie Informationen zu Ausbildung, Schulbildung, Einkommen und Beruf des Partners erstellen. Es sind ebenfalls Informationen zur Schulbildung, Ausbildung und Studium der Kinder vorhanden sowie das Auszugsdatum, Kontakthäufigkeit und Fragen zu den Enkelkindern. Es bleibt anzumerken, dass es sich bei dieser Stichprobe um eine stark bildungsselektive Teilpopulation handelt, deren Selektivität im Verlauf von 40 Jahren noch etwas zugenommen hat (Birkelbach 2011: 27).

Für die folgenden Analysen wurden nicht alle Befragten einbezogen, sondern nur diejenigen, die im Alter von 56 Jahren verheiratet sind, Kinder haben und die keine Trennung, Scheidung oder Verwitwung erlebt haben. Es handelt sich somit um Personen, die den Normalverlauf des Familienzyklus (Glick 1955; Höhn 1982) durchlebt haben und deren Familienverlauf durch die gleichen Ereignisse geprägt ist und zwar von einer Eheschließung, der Geburt und dem Auszug der Kinder. Das rigorose Aussortieren von Personen mit kritischen Ereignissen im Familienverlauf (Filipp 1990) soll verhindern, dass diese sich auf den Eintritt in die nachelterliche Phase auswirken. Es sollen hauptsächlich die Einflüsse der biographischen Merkmale der Eltern und Kinder ausgemacht werden. Welchen Einfluss kritische Ereignisse auf den Eintritt in die nachelterliche Phase besitzen, müsste in einer gesonderten Arbeit untersucht werden. Immerhin erfüllen insgesamt 605 Personen von den 1301 Befragten, also 47 \% der Stichprobe, diese Kriterien. Bei diesen 605 Befragten wurde überprüft, zu welchem Zeitpunkt das letzte Kind aus dem elterlichen Haushalt ausgezogen ist. Die genaue Fragestellung lautete: „In welchem Jahr hatte $\mathrm{Ihr}$ Kind zum ersten Mal einen eigenen Hausstand, ich meine ein eigenes Zuhause?" Dabei muss es sich nicht zwangsläufig um das jüngste Kind handeln.

\subsection{Methode}

Um den Übergang in die nachelterliche Phase im Lebensverlauf verorten zu können, wird mit der Sterbetafelmethode eine Übergangsrate geschätzt. Die Übergangsrate entspricht dem Risiko den momentanen Zustand zu wechseln, z.B. von ledig zu verheiratet (Diekmann/Mitter 1984). Die Übergangsrate $h_{j k}(t)$ gibt die Wahrscheinlichkeit an, dass in einem bestimmten Zeitintervall $t$ ein Ereignis eintritt, unter der Voraussetzung, dass es bis zum Beginn dieses Intervalls noch nicht eingetreten ist und kann wie folgt beschrieben werden (Blossfeld 2010: 1001):

$$
h_{j k}(t)=P(T=t \mid T \geq t) t=1,2,3 \ldots
$$


Dabei werden die Verweildauern in Monatsintervalle eingeteilt und „für jedes dieser Intervalle werden die Anzahl der Untersuchungseinheiten, die am Beginn des Intervalls noch dem Ereignisrisiko ausgesetzt sind, die Anzahl der Untersuchungseinheiten, die im Intervall ein Ereignis erfahren, und die Anzahl der Untersuchungseinheiten, die im Intervall zensiert werden, gezählt." (Blossfe/d 2010: 1003) Daraus ergibt sich die Wahrscheinlichkeit, dass vor einem bestimmten Zeitpunkt kein Ereignis eintritt, hier also dass der Übergang in die nachelterliche Phase nicht stattfindet. Für die grafische Darstellung der Überlebensquote wird der kumulierte Anteil der Überlebenden am Intervallende verwendet und somit kann abgelesen werden, in welchem Alter welcher Anteil der Befragten dem Risiko des Übergangs ausgesetzt ist.

Anschließend wird mittels einer Cox-Regression (Cox 1972; Blossfeld et al. 2007; Blossfeld 2010) geprüft, welche Faktoren den Eintritt in die nachelterliche Phase beschleunigen bzw. verlangsamen. Es wird dabei das semiparametrische Modell der Cox-Regression angewendet, also ein ereignisanalytisches Verfahren, welches in der Literatur häufig als Proportional-Hazards-Modell bezeichnet wird (Blossfeld 2010). Dieses bietet den Vorteil, dass es angewendet werden kann, auch wenn nicht alle Personen während der Beobachtungszeit den Auszug des letzten Kindes erlebt haben. Darüber hinaus eignet es sich besonders gut bei Analysen, die keine begründbaren Annahmen über den Verlauf der Basisratenfunktion besitzen (Weick 1993: 97), was für den Eintritt in die nachelterliche Phase gilt. Die untersuchten Zeitspannen beginnen im Alter von 30 Jahren und enden mit dem Ereignis des Auszugs des letzten Kindes, oder bei rechtszensierten Fällen mit dem Alter bei der Befragung.

Bei der ereignisanalytischen Regression bildet die Rate des Auszugs des letzten Kindes die abhängige Variable. Es handelt sich um die Wahrscheinlichkeit, dass Eltern, die zu einem bestimmten Zeitpunkt noch mit Kindern im Haushalt leben, in die nachelterliche Phase eintreten. Die Eintrittsrate $r(t)$ wird dabei durch eine für alle Probanden gleiche Übergangsratenfunktion $h_{0}(t)$ und die mit ihr multiplikativ und in exponentieller Form verbundenen Kovariateneinflüsse exp(.) dargestellt (Diekmann/Mitter 1993):

$$
r(t)=h_{o}(t) \cdot \exp \llbracket\left(\beta_{1} x_{1}+\beta_{2} x_{2}+\cdots \rrbracket \beta_{m} x_{m}\right)
$$

Die Schätzung des Cox-Modells erfolgt durch die Partial-Likelihood-Methode (Blossfeld 2010: 1005). Bei der ereignisanalytischen Regression interessieren vornehmlich die Effekte der Kovariaten auf das Eintrittsrisiko. Diese lassen sich an den $\beta$-Koeffizienten ablesen, wobei die Antilogarithmen der $\beta$-Koeffizienten $\left(\alpha_{i}=\exp \beta_{i}\right)$ anschaulicher sind, denn diese lassen sich als Prozenteffekte $\left(\alpha_{i}-1\right) \cdot 100$ der Kovariaten auf das Eintrittsrisiko interpretieren (Berger 2009; Ziegler/Schladt 1993).

\subsection{Daten: Kovariaten der Cox-Regression}

Aufbauend auf den bisherigen Forschungsergebnissen (siehe Abschnitt 2) lassen sich mit den Daten des KGP die folgenden unabhängigen Variablen in die Analysen 
mit einbeziehen. Darüber hinaus werden zusätzliche Faktoren aufgenommen, die ebenfalls als mögliche Einflussgrößen ausgemacht werden können.

Privatbiografie der Eltern (befragte): Das Heiratsalter (in Jahren), das Alter bei der Geburt des ersten Kindes (in Jahren) und die Kinderanzahl werden als wichtige Einflüsse benannt. Bei allen drei Variablen ist anzunehmen, dass je geringer sie sind, desto eher erfolgt der Eintritt in die nachelterliche Phase. Zusätzlich kann der Abstand der Geburten als erklärende Variable aufgenommen werden, denn je größer der Abstand zwischen dem ersten und dem letzten Kind, desto später liegt der Eintritt. Dieser Abstand wird in Jahren als Differenz zwischen den Zeitpunkten der ersten und der letzten Geburt dargestellt. Darüber hinaus soll geprüft werden, wie sich das Alter der Eltern bei ihrem eigenen Auszug (in Jahren) auf die Eintrittswahrscheinlichkeit in die nachelterliche Phase auswirkt. Denn ein früher eigener Auszug der Eltern kann einen positiven Einfluss auf den Auszugszeitpunkt der Kinder ausüben, weil er als ein gelungener Start in die Unabhängigkeit gesehen wird, oder aber einen negativen, weil er eher mit Belastungen und Unsicherheiten verbunden wird und diese Erfahrungen von den Eltern an die eigenen Kinder herangetragen werden.

Bildungs- und Berufsbiografie der Eltern (befragte): Das Bildungsniveau wird durch das Erreichen des Abiturs und eines Studienabschlusses abgebildet. Da im KGP ebenfalls Fragen zur Bildungsbiografie der Partner gestellt wurden, können der Schul- und Hochschulabschluss für die Partner konstruiert werden. Dabei wird zwischen Müttern und Vätern unterschieden, also separate Variablen für die Mütter und Väter mit Abitur und Universitätsabschluss erstellt. Es wird davon ausgegangen, dass ein höherer Abschluss den Eintritt in die nachelterliche Familienphase verzögert. Die Berufsausbildung wird ebenfalls aufgenommen. Den Einfluss einer Ausbildung zu bestimmen, gestaltet sich schwerer, denn zum einen könnten sich Eltern, die eine Ausbildung gemacht haben, für ihre Kinder einen höherwertigeren Bildungsabschluss vorstellen oder aber mit einem gleichwertigen zufrieden sein und sich weigern, ihren Kindern ein Studium zu finanzieren. Da sich in den Forschungsergebnissen die Berufstätigkeit der Mutter als signifikant erwies, wird die zeitabhängige Variable ,Erwerbstätigkeit der Mutter zum Zeitpunkt des Auszugs des Kindes' erstellt.

Beim Einkommen wurde das monatliche Haushaltseinkommen gewählt, welches sich aus dem höchsten erreichten Einkommen in der Phase zwischen dem Alter von 43 und 56 Jahren und dem aktuellen Partnereinkommen berechnet. Mit der Verwendung des höchsten erreichten Einkommens kann gegenüber dem Durchschnittseinkommen auf mehr Varianz zurückgegriffen werden. Es gilt zu bestimmen, in welche Richtung das Einkommen auf den Eintritt in die nachelterliche Phase wirkt. Da im KGP nicht nach Wohnverhältnissen gefragt wurde, lässt sich keine eindeutige Unterscheidung treffen, ob das Einkommen die haushaltsspezifischen oder die Möglichkeit von transferierbaren Ressourcen abbildet.

Die Biografie des Kindes der Befragten lässt sich nicht so einfach wie die der Eltern integrieren. Denn dadurch, dass die Personen mehrere Kinder haben, können sich signifikante Effekte gegenseitig aufheben, wenn bspw. jemand einen Sohn und eine Tochter hat, kann das Einbeziehen des Geschlechts alleine keine eindeutigen Effekte 
aufweisen, wenn Töchter früher und Söhne später ausziehen. Deshalb wurden die Variablen für die Kinder auf Basis des letzten ausgezogenen Kindes erstellt bzw. des jüngsten noch im Haushalt verbliebenen Kindes. Darüber hinaus wurden keine Zeitpunkte der Kinderbiografie erfragt, somit lassen sich keine Aussagen über den zeitlichen Zusammenhang vom Eintritt in die nachelterliche Phase und bspw. der Erwerbstätigkeit machen. Es wurden fünf Dummyvariablen erstellt: Abitur (ja/nein), Hochschulabschluss (ja/nein), Berufsausbildung abgeschlossen (ja/nein), Erwerbstätigkeit (ja/nein), Geschlecht (männlich/weiblich). Es werden nur diejenigen Kinder in die Analysen aufgenommen, die 18 Jahre und älter sind, da im Gymnasiastenpanel die Eltern nur für jedes Kind, das über 18 Jahre ist, gefragt wurden, ob es einen eigenen Hausstand hat.

Im Modell in Abbildung 1 werden die erwähnten Variablen und ihr vermuteter Einfluss auf den Eintritt in die nachelterliche Phase dargestellt, jeweils differenziert nach Merkmalen der Eltern und Kinder.

Abb. 1: Kovariateneffekte auf das Eintrittsrisiko in die nachelterliche Phase nach Merkmalen der Eltern und Kinder nach vermuteter Wirkrichtung

Eltern
Kind

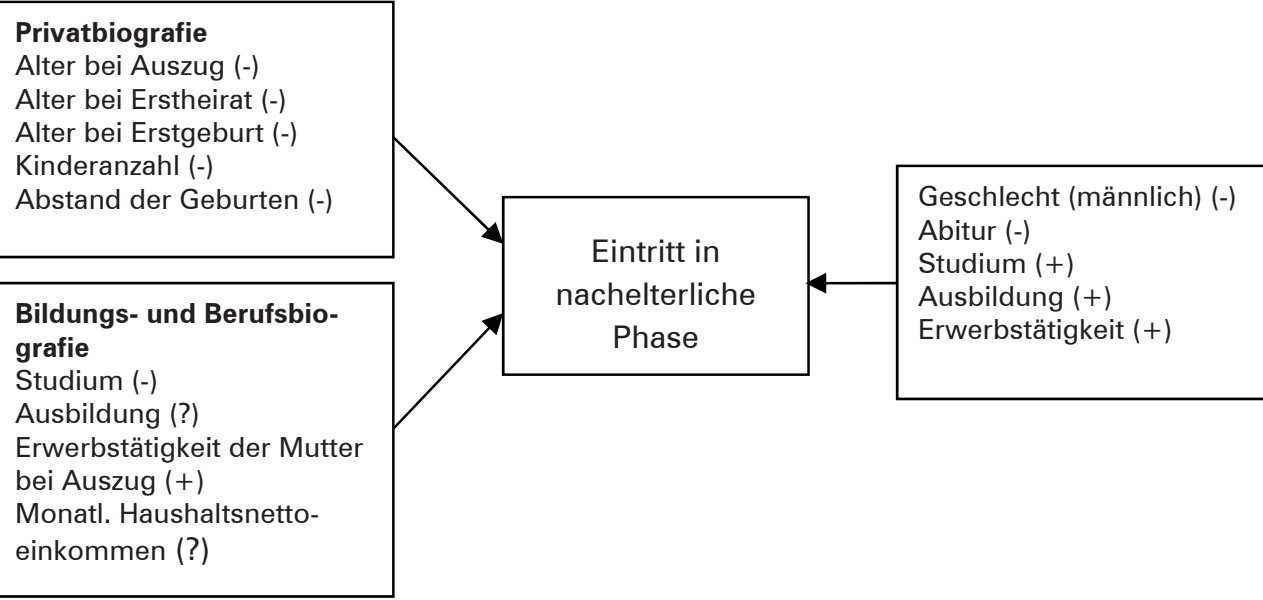

Quelle: eigene Darstellung

In Tabelle 1 sind die genannten Variablen samt Wertebereich und Mittelwerte dargestellt. Sie werden an dieser Stelle nur kurz aufgeführt, um die Stichprobe zu präsentieren und um ein besseres Verständnis für die Effekte dieser Variablen in der Cox-Regression zu vermitteln.

Die Befragten des KGP weisen eine überdurchschnittlich hohe Bildung auf. So haben 69 \% der Väter und 53 \% der Mütter einen Studienabschluss, während sich 
Tab. 1: Übersicht der unabhängigen Variablen: Wertebereich, Mittelwerte und Standardabweichungen für Eltern und Kinder über 18 Jahre $(N=530)$

\begin{tabular}{lccr}
\hline Variable & Wertebereich & Mittelwert & SD \\
\hline Alter bei Eintritt in nachelterliche Phase & $43-59$ & 54.6 & 2.81 \\
Eltern (Befragte) & & & \\
Erstheiratsalter & $18.9-39.3$ & 27.5 & 3.95 \\
Erstelternschaft & $20-42.5$ & 30.3 & 3.91 \\
Alter bei eigenem Auszug & $17.7-36.1$ & 23.3 & 3.24 \\
Kinderanzahl & $1-5$ & 2.2 & 0.85 \\
Abstand der Geburten in Jahren & $0-13.7$ & 3.8 & 3.04 \\
Berufsausbildung Befragte/r & $0 / 1$ & .39 & - \\
Studium Vater & $0 / 1$ & .69 & - \\
Studium Mutter & $0 / 1$ & .53 & - \\
Abitur Vater & $0 / 1$ & .78 & - \\
Abitur Mutter & $0 / 1$ & .65 & 8102.1 \\
Monatl. Haushaltsnettoeinkommen & $400-129294$ & 6282.50 & - \\
Erwerbstätigkeit der Mutter beim Auszug & & & \\
(zeitabhängig) & $0 / 1$ & .731 & - \\
Kinder & & & - \\
Geschlecht (männlich) & $0 / 1$ & .51 & - \\
Abitur & $0 / 1$ & .67 & - \\
Berufsausbildung & $0 / 1$ & .25 & - \\
Studium & $0 / 1$ & .58 & - \\
Erwerbstätigkeit & $0 / 1$ & .29 & - \\
Alter & $18.1-36.9$ & 22.20 & - \\
\hline
\end{tabular}

1 Dieser Anteil bezieht sich nur auf die Frauen in der Stichprobe. Für die gesamte Stichprobe beträgt der Anteil der arbeitenden Mütter $33 \%$.

Quelle: eigene Berechnungen

in der allgemeinen Bevölkerung in der Altersgruppe der 50- bis 59-Jährigen durchschnittlich nur $17 \%$ mit Studium befinden (Datenreport 2011). Ebenfalls sehr hoch ist der Anteil der Abiturienten unter den Eltern, mit $78 \%$ bei den Vätern und $65 \%$ bei den Müttern und gerade mal $24 \%$ in der allgemeinen Bevölkerung der gleichen Altersgruppe. Bei der Berufsausbildung hingegen weisen die ehemaligen Gymnasiasten einen geringeren Anteil aus als die Vergleichsgruppe in der Gesamtbevölkerung mit $58 \%$. Das Haushaltsnettoeinkommen weist eine breite Streuung auf und sein Durchschnitt liegt bei über 6000 Euro. Es arbeiten $73 \%$ der Mütter zum Zeitpunkt des Auszugs des letzten Kindes.

Bei den Kindern findet sich, wie bei den Eltern auch, ein hoher Anteil mit Abiturabschluss wieder (67\%), während nur $25 \%$ eine Ausbildung abgeschlossen ha- 
ben. Dafür besitzen bereits $58 \%$ einen Hochschulabschluss und $29 \%$ gehen einer hauptberuflichen Tätigkeit nach.

Bevor die unabhängigen Variablen in die Cox-Regression aufgenommen werden, sollen zuerst mögliche Korrelationen zwischen den einzelnen Faktoren ausfindig gemacht und beseitigt werden, um mögliche Multikollinearitäten auszuschließen. Vor allem bei den demografischen Größen ist damit zu rechnen, dass diese sich gegenseitig beeinflussen. Denn wer früh heiratet, wird höchstwahrscheinlich auch in einem jüngeren Alter Kinder bekommen als jemand, der spät heiratet. Bei Variablen, die zu hoch miteinander korrelieren $(r>0.3)$ wird eine davon aus den Analysen ausgeschlossen. Die Ergebnisse der Korrelationen sind nicht dargestellt, die wichtigsten Erkenntnisse, die für das Vorgehen der weiteren Analyse von Bedeutung sind, werden aber kurz erwähnt: das Erstheiratsalter korreliert sehr stark mit dem Alter bei der Geburt des ersten Kindes, es wird aber erwartet, dass das Alter bei der Geburt stärker mit dem Auszug der Kinder zusammenhängt als das Alter bei der Heirat. Die Kinderanzahl und der Geburtenabstand korrelieren sehr hoch, da aber die Anzahl der Kinder in anderen Umfragen öfter erhoben wird als der gesamte Privatlebenslauf, wird diese Variable einbezogen. Die Bildungsvariablen korrelieren ebenfalls alle sehr hoch miteinander, weshalb für Mütter, Väter und Kinder jeweils nur der Studienabschluss als unabhängige Variable genommen wird, da die Anteile derer ohne Studienabschluss höher sind als ohne Abitur.

\section{Die zeitliche Positionierung des Übergangs in die nachelterliche Phase im Lebensverlauf}

Ab wann können Eltern damit rechnen ihre eigenen vier Wände wieder zu zweit bewohnen zu können? Diese Frage wird mittels der "Überlebensquote" für Eltern mit Kindern im Haushalt grafisch dargestellt. Von den 605 Personen befinden sich 254 (42\%) Personen in der nachelterlichen Phase und somit ohne Kinder im Haushalt und bei 351 (58 \%) Personen lebt mindestens noch ein Kind im Haushalt. Lauterbach (2004) datiert den Zeitpunkt des Eintritts in die nachelterliche Phase auf die sechste Lebensdekade (Lauterbach 2004: 162). So errechnen z.B. Mayer und Wagner (1989) den Median des Alters für Väter der Kohorte 1949-51 auf 56 Jahre, falls diese Söhne haben, und auf 52 Jahre, falls nur Töchter betrachtet werden. Für Mütter desselben Jahrgangs ergibt sich ein Median von 53 Jahren bei Söhnen und 49 Jahren bei Töchtern. Bei der hier untersuchten Kohorte sind die Unterschiede zwischen Eltern mit Töchtern und Söhnen nicht so hoch: hier liegt der Median von Vätern bei 57 Jahren für Töchter und 58 Jahren für Söhne und von Müttern bei 55 Jahren für Töchter und 57 Jahren für Söhne. ${ }^{2}$ Werden unterschiedliche Kohorten betrachtet, so konstatiert Lauterbach (2004: 166-175), dass Väter und Mütter seit der Nachkriegszeit immer früher in die späte Familienphase eintreten und der Anteil derjenigen, die diese Phase überhaupt erleben, angestiegen ist.

2 Da nicht alle Eltern den Auszug des letzten Kindes erlebten, wurde der Median mit der Sterbetafelmethode geschätzt. 
Der zeitliche Unterschied beim Eintritt in die nachelterliche Phase zwischen Männern und Frauen lässt sich nicht nur durch den Median bestimmen, er lässt sich ebenfalls an den Überlebenskurven für Männer und Frauen erkennen (Abb. 2). Die beiden Kurven unterscheiden sich signifikant voneinander (Wilcoxon: 17,78 $p=.000$; Log Rank: 15,16 $p=.000$ ). Frauen erleben somit den Eintritt in die nachelterliche Phase früher als Männer. Im Alter von 56 Jahren sehen sich bereits $53 \%$ der Mütter und nur $38 \%$ der Väter mit einem kinderlosen Haushalt konfrontiert. Die Überlebenskurven verlaufen aber parallel zueinander, so dass die Männer mit etwa drei Jahren Abstand den Frauen folgen. Dieser Abstand entspricht recht genau dem für diese Kohorte errechneten früheren Eintritt der Frauen in den Familienzyklus: Bei der hier betrachteten Kohorte beträgt das durchschnittliche Erstheiratsalter für Männer 29 Jahre und für Frauen 26 Jahre.

Anhand der Überlebenskurve kann man zusätzlich erkennen, dass bei Frauen frühestens mit etwa 45 Jahren und bei Männern mit 48 Jahren mit dem Auszug des letzten Kindes zu rechnen ist und sich dann allmählich der Anteil erhöht. Der Übergang vollzieht sich für die Hälfte der Eltern zwischen dem 45. und 55. (Mütter) bzw. 48. und 58. Lebensjahr (Väter). Das bedeutet, dass ein Großteil der Eltern bereits vor Eintritt in den Ruhestand in einem kinderlosen Haushalt wohnt. Da es sich beim Gymnasiastenpanel um eine positiv bildungsselektierte Teilpopulation handelt, ist es wahrscheinlich, dass die Überlebensquote bei der Gesamtbevölkerung bereits in einem früheren Lebensalter sinkt.

Abb. 2: „Überlebensquote“ von Müttern und Vätern im Haushalt mit Kindern

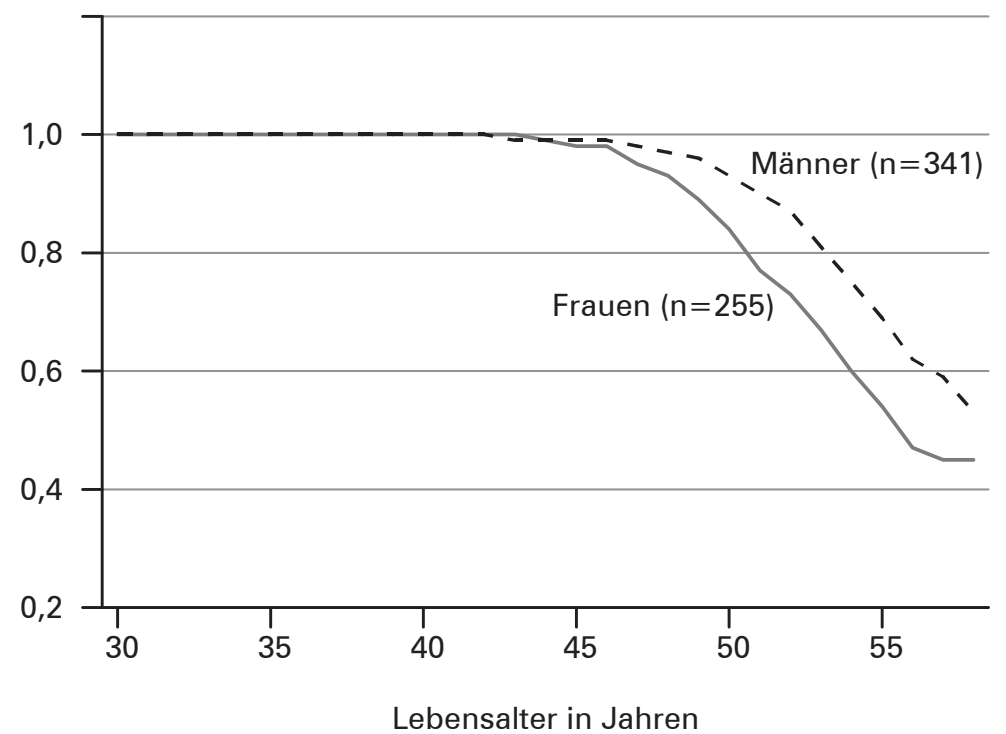

Quelle: eigene Berechnungen und Darstellung 


\section{Der Eintritt in die nachelterliche Phase}

An der zuvor dargestellten zeitlichen Positionierung des Eintritts in die nachelterliche Phase lässt sich sehr gut erkennen, wann Eltern mit dem Auszug ihres letzten Kindes rechnen können. In diesem Abschnitt sollen nun die in Abschnitt 3.3 genannten Variablen als mögliche Einflüsse auf die Wahrscheinlichkeit des Eintritts in die nachelterliche Phase in die Cox-Regression aufgenommen werden. Die Ergebnisse finden sich in Tabelle 2. Dabei wurden unterschiedliche Modelle gerechnet, in denen die Einflüsse der Bereiche der Privat- und Erwerbsbiografie sowie die Merkmale der Kinder zum einen separat und zum anderen in einem Gesamtmodell errechnet wurden.

Die Privatbiografie der Eltern wirkt sich sehr stark auf das Eintrittsrisiko in die nachelterliche Familienphase aus, wie in Modell 1 zu sehen ist. Das Alter bei der ersten Geburt senkt das Eintrittsrisiko signifikant um $19 \%$, dieser Wert ergibt sich durch die Subtraktion des $\alpha$-Koeffizienten (.81) vom Wert 1. Er bedeutet, dass pro zusätzliches Jahr bei der Geburt des ersten Kindes, die Hazardrate um $19 \%$ gesenkt wird, der Eintritt in die nachelterliche Phase sich somit verlangsamt. Einen ebenfalls sehr starken Einfluss besitzt die Kinderanzahl: pro zusätzlichem Kind senkt sich das Eintrittsrisiko um $61 \%$. Auch das Alter beim eigenen Auszug verlangsamt den Eintritt: je älter die Befragten bei ihrem eigenen Auszug sind, desto später treten sie in die nachelterliche Phase ein. Da diese Variable trotz Kontrolle des Alters bei der Geburt des ersten Kindes einen signifikanten Effekt auf dem 5-Prozent-Niveau aufweist, scheint die Erfahrung des eigenen Auszugs einen eigenständigen Effekt zu besitzen. Da das Auszugsalter mit der Bildungsbiografie zusammenhängt (Abschnitt 3.3) ist zu vermuten, dass dieses an Signifikanz verliert, wenn die Bildungsbiografie der Eltern ebenfalls betrachtet wird. Der starke Einfluss der privaten Lebensbiografie auf das Eintrittsrisiko in die nachelterliche Phase ist aber nicht sonderlich überraschend. Interessanter sind die Effekte der anderen Kovariaten unter Kontrolle der privaten Lebensbiografie.

Bei Modell 2 wird neben der privaten Lebensbiografie auch die Bildungs- und Erwerbsbiografie der Eltern einbezogen. Zum einen ist zu erkennen, dass die starken Einflüsse des Alters bei der Geburt des ersten Kindes und die Kinderanzahl bestehen bleiben bzw. sich sogar leicht erhöhen, zum anderen, dass das Alter beim eigenen Auszug immer noch einen signifikanten Effekt hat, obwohl für die Bildungsbiografie kontrolliert wird. Dies lässt vermuten, dass Eltern, die selber spät ausgezogen sind, ihre eigenen Kinder ebenfalls länger bei sich wohnen haben. Bei der Bildungs- und Berufsbiografie zeigt sich, dass weder der Hochschulabschluss des Vaters, eine Ausbildung oder die Erwerbstätigkeit der Eltern, insbesondere der Mutter, bei Auszug der Kinder sich auf die Eintrittsgeschwindigkeit in die nachelterliche Phase auswirken. Es macht also keinen Unterschied bei der Auszugsentscheidung der Kinder, ob die Mutter zum Zeitpunkt des Auszugs arbeitet oder nicht. Dafür treten aber Mütter mit einem Hochschulabschluss schneller in die Phase ein als Mütter ohne Studienabschluss und zwar unabhängig von der Kinderzahl und des Erstgeburtsalters. Die Rate erhöht sich dadurch um 36 \%. Ein Studium bewirkt zwar in der Regel ein höheres Erstgeburtsalter, aber es führt offensichtlich auch zu einem 


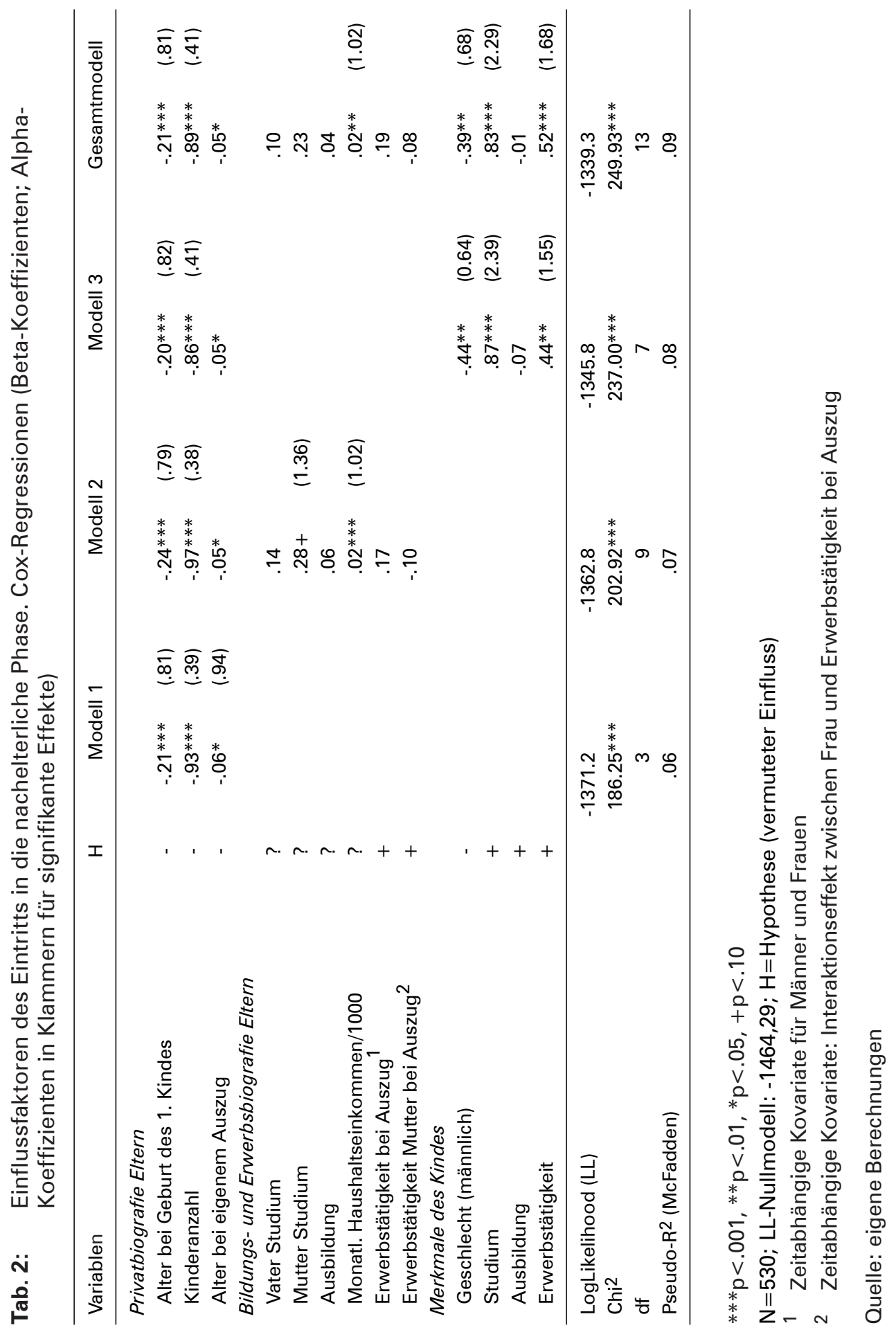


früheren Eintritt in die nachelterliche Phase. Dass sich ein Hochschulabschluss der Mutter stärker auf die Auszugsgeschwindigkeit auswirkt als der des Vaters kann evtl. darauf zurückgeführt werden, dass Mütter durch ihren intensiveren Kontakt zu ihren Kindern sie in ihrer Bildungslaufbahn mehr beeinflussen und sie eher zu einem Studium bewegen können, womit meistens auch ein Umzug verbunden ist. Das monatliche Haushaltseinkommen (geteilt durch 1000) besitzt ebenfalls einen positiven und signifikanten Effekt. Somit lässt sich festhalten, dass ein hohes Einkommen den Auszug der eigenen Kinder beschleunigt, da diese Eltern ihren Kindern vermutlich eher die Möglichkeit bieten können einen eigenen Hausstand zu gründen. Insgesamt lässt sich festhalten, dass sich der Einfluss des Bildungs- und Berufslebens zwar auf die Ereignisse der Familiengründung erstreckt (Birkelbach 1998), aber er verliert offenbar an Bedeutung für die Folgephasen des Familienzyklus.

In Modell 3 wurden die Merkmale der Kinder in die Analysen aufgenommen. Diese Ergebnisse sind allerdings mit einiger Vorsicht zu betrachten, denn wie schon erwähnt, handelt es sich nicht um zeitabhängige Variablen. Somit ist bspw. ein kausaler Zusammenhang vom Eintritt in die Erwerbstätigkeit und dem Auszugszeitpunkt nicht nachvollziehbar. Da als Basis die Daten des letzten ausgezogenen Kindes in Vergleich zum jüngsten noch im Haushalt lebenden Kind dienen, lassen sich die Ergebnisse aus Modell 3 wie folgt interpretieren: Ist das letzte zuhause lebende Kind ein Sohn, so senkt sich das Eintrittsrisiko in die nachelterliche Phase um $36 \%$. Besitzt es einen Studienabschluss, beschleunigt dies sogar den Eintritt in die nachelterliche Phase um $139 \%$ (Alpha-Koeffizienten > 1 sind als Prozentzahlen zu lesen). Ist das Kind erwerbstätig, erhöht sich das Eintrittsrisiko um $55 \%$. Eine Ausbildung hingegen besitzt keinen signifikanten Effekt. Diese Ergebnisse decken sich mit denjenigen Studien, die das Auszugsverhalten von Männern und Frauen untersucht haben (z.B. Ziegler/Schladt 1993; Berger 2009; Lauterbach 2004).

Im Gesamtmodell werden die Einflüsse der einzelnen Variablen unter der Kontrolle und Konstanthaltung der anderen Kovariaten berechnet. Der starke Einfluss der Privatbiografie bleibt dabei bestehen. Bei der Bildungs- und Erwerbsbiografie hingegen verliert der Studienabschluss der Mutter unter Kontrolle der Kindermerkmale an Signifikanz, aber nur wenig von seiner Effektstärke. Das Haushaltseinkommen behält den schwachen positiven Einfluss. Die Effekte der Kindermerkmale verändern sich nur geringfügig, so erhöht das Studium des Kindes die Rate um 129 \% und die Erwerbstätigkeit um $68 \%$ und Söhne verlangsamen den Übergang um $32 \%$. Die Merkmale der Kinder wirken sich offenbar stärker auf die Eintrittswahrscheinlichkeit in die nachelterliche Phase aus als die der Eltern. Nur das Einkommen besitzt weiterhin einen schwachen positiven Effekt auf den Eintritt

Durch den Vergleich der Log-Likelihood-Werte sowie des darauf basierenden Kennwertes Pseudo- $\mathrm{R}^{2}$ wird deutlich, dass die Hinzunahme der Bildungs- und Berufsvariablen keine Verbesserung des Modells bewirkt. Besser angepasst wird das Modell aber durch die Kindermerkmale, wohingegen das Gesamtmodell keine Verbesserung bewirkt. Für die Analyse des Eintrittsrisikos in die nachelterliche Phase reichen die Angaben der Privatbiografie und der Kindermerkmale offenbar aus. 


\section{$6 \quad$ Fazit}

Der Auszug des letzten Kindes wird vom Großteil der Eltern in der sechsten Lebensdekade erlebt. Jedoch zeigen die durchgeführten Analysen, dass für diejenigen, die früh aus dem eigenen elterlichen Haushalt ausgezogen sind, der Eintritt in die nachelterliche Phase früher erfolgt als für diejenigen, die später ausgezogen sind. Einen noch größeren Einfluss auf den Eintrittszeitpunkt besitzt, wie zu erwarten, das Alter bei der Geburt des ersten Kindes: Je jünger die Eltern sind, desto früher können sie mit dem Auszug der Kinder rechnen. Ebenso bedeutend ist die Kinderanzahl, ist diese gering, sind die Eltern früher allein im Haushalt. Damit steht fest: Der Eintritt in die Nach-Elternphase hängt stark von den Entscheidungen im frühen, privaten Lebensverlauf ab.

Der berufliche Lebensweg der Eltern spielt keine Rolle für ihren Eintritt in die nachelterliche Phase. Weder ein Studienabschluss, noch eine Ausbildung oder eine Erwerbstätigkeit der Mutter zum Zeitpunkt des Auszugs können als eindeutige Faktoren ausgemacht werden. Lediglich ein höheres Einkommen beschleunigt den Eintritt und somit den Auszug des letzten Kindes. Wesentlich stärker von Bedeutung ist die Berufs- und Bildungsbiografie der Kinder. Bei Kindern, die studieren oder erwerbstätig sind, können die Eltern viel früher mit einem kinderlosen Haushalt rechnen. Ebenso bedeutend ist das Geschlecht des Kindes: bei Söhnen zögert sich die Phase des gemeinsamen Haushalts länger hinaus als bei Töchtern.

Die Längsschnittanalyse zeigt deutlich, dass für den Eintritt in die nachelterliche Phase das Privatleben der Eltern und die Merkmale der Kinder von Bedeutung sind.

Es wurden nicht alle anfangs erwähnten Determinanten in die Analysen mit aufgenommen, da diese teilweise sehr stark miteinander korrelierten und dieser Effekt sich im Regressionsmodell niedergeschlagen hätte. Für Folgeanalysen lässt sich aber festhalten, dass die korrelierenden Variablen untereinander ausgetauscht werden können, da sie den gleichen Effekt besitzen.

Abschließend soll auch auf einige Limitationen dieser Untersuchung eingegangen werden. Die Ergebnisse erfolgten auf Basis einer bildungsselektierten Stichprobe und sind nicht auf die Gesamtpopulation übertragbar. Zudem sind eingangs einige Einschränkungen bzgl. des Familienverlaufs gemacht worden: Es wurden nur Personen in die Analysen einbezogen, die einen normalen Familienverlauf ohne Scheidung oder Verwitwung aufweisen. Einflüsse dieser Ereignisse müssten genauer theoretisch begründet und empirisch belegt werden. Darüber hinaus handelt es sich nur um eine Geburtskohorte, interessant wäre es auch zu erfahren, ob sich die hier gezeigten Einflüsse bei anderen Jahrgängen ebenfalls als signifikant erweisen.

Von Interesse für weitere Analysen zum Übergang in die nachelterliche Phase wäre auch die Beziehungsqualität zwischen Eltern und Kindern vor dem Auszug, die im KGP nicht erfragt wurde. Darüber hinaus könnte die Wohnsituation der Familien und damit einhergehend der Wohnkomfort zusätzliche Erkenntnisse über das Eintrittsrisiko liefern. In weiteren Analysen sollte genauer untersucht werden, wie sich der Eintritt selber auf das Leben der Eltern auswirkt, so könnte es sein, dass sich der Auszug des letzten Kindes auf die Bedeutung der Familie auswirkt, indem 
er ihren Stellenwert senkt. Oder aber der Wegfall der Betreuung der Kinder könnte sich auf das Berufsleben der Eltern auswirken, insbesondere der Mütter, da ihnen nun mehr Zeit für eine berufliche Tätigkeit zur Verfügung steht. Ebenso könnte der Eintritt in die nachelterliche Phase andere Lebensbereiche beeinflussen, wie z.B. die Freizeitgestaltung.

\section{Literatur}

Bart, Pauline B. 1971: Depression in middle-aged women. In: Gornick, Vivian; Moran, Barbara K. (Hrsg.): Woman in sexist society. Studies in power and powerlessness. New York: Basic Books: 99-117.

Berger, Fred 2009: Auszug aus dem Elternhaus - Strukturelle, familiale und persönlichkeitsbezogene Bedingungsfaktoren. In: Fend, Helmut (Hrsg.): Lebensverläufe, Lebensbewältigung, Lebensglück. Ergebnisse der LifE-Studie. Wiesbaden: VS Verlag für Sozialwissenschaften: 195-243.

Birkelbach, Klaus W. 1998: Berufserfolg und Familiengründung. Lebensläufe zwischen institutionellen Bedingungen und individueller Konstruktion. Opladen: Westdeutscher Verlag.

Birkelbach, Klaus W. 2011: Ausfälle im Kölner Gymnasiastenpanel 1969 - 2010: Ursachen und mögliche Folgen für die Datenqualität. In: Birkelbach, Klaus W. et al. (Hrsg.): Vor dem Lebensabend - eine dritte Wiederbefragung zu Lebenserfolg und Erfolgsdeutung ehemaliger 16-jähriger Gymnasiasten im 56. Lebensjahr: Erste Analysen. Unveröffentlichter Zwischenbericht zur Vorlage bei der Deutschen Forschungsgemeinschaft. Köln/Essen: 1-30.

Blossfeld, Hans; Golsch, Katrin; Rohwer, Götz 2007: Event History Analysis with Stata. Mahwah (NJ): Erlbaum.

Blossfeld, Hans 2010: Survival- und Ereignisanalyse. In: Wolf, Christof; Best, Henning (Hrsg.): Handbuch sozialwissenschaftliche Datenanalyse. Wiesbaden: VS Verlag für Sozialwissenschaften: 995-1016.

Cox, David R. 1972: Regression Models and Life-Tables. In: Journal of the Royal Statistical Society. Series B, 34: 187-220.

Datenreport 2011: Ein Sozialbericht für die Bundesrepublik Deutschland (2011). Bonn: $\mathrm{BpB}$, Bundeszentrale für politische Bildung.

Dennerstein, Lorraine; Dudley, Emma; Guthrie, Janet 2002: Empty nest or revolving door? A prospective study of women's quality of life in midlife during the phase of children leaving and re-entering the home. In: Psychological Medicine 32: 545-550 [doi: 10.1017/S0033291701004810].

Deykin, Eva; Jacobson, Shirley; Klerman, Gerald; Solomon, Maida 1966: The empty nest: Psychosocial aspects of conflict between depressed women and their grown children. In: American Journal of Psychiatry 122: 1422-1426.

Diekmann, Andreas; Mitter, Peter 1984: Methoden zur Analyse von Zeitverläufen: Anwendungen stochastischer Prozesse bei der Untersuchung von Ereignisdaten. Stuttgart: Teubner.

Diekmann, Andreas; Mitter, Peter 1993: Methoden der Ereignisanalyse in der Bevölkerungssoziologie: Stand und Probleme. In: Diekmann, Andreas; Weick, Stefan (Hrsg.): Der Familienzyklus als sozialer Prozess. Bevölkerungssoziologische Untersuchungen mit den Methoden der Ereignisanalyse. Berlin: Duncker \& Humblot: 20-65. 
Feser, Herbert; Müller-Daehn, Sigrid; Schmitz, Uta 1989: Familienfrauen im mittleren Alter. Lebenssituation und Zukunftsperspektiven. Stuttgart: W. Kohlhammer.

Filipp, Sigrun-Heide (Hrsg.) 1990: Kritische Lebensereignisse. 2., erw. Auflage München: Psychologie Verlags Union.

Glick, Paul C. 1955: The Life Cycle of the Family. In: Marriage and Family Living 17,1: 3-9 [http://www.jstor.org/stable/346771, 27.01.2015].

Höhn, Charlotte 1982: Der Familienzyklus. Zur Notwendigkeit einer Konzepterweiterung. Boppard am Rhein: Boldt.

Lauterbach, Wolfgang 2004: Die multilokale Mehrgenerationenfamilie. Zum Wandel der Familienstruktur in der zweiten Lebenshälfte. Würzburg: Ergon.

Mayer, Karl-Ulrich; Wagner, Michael 1989: Wann verlassen Kinder das Elternhaus? In: Strohmeier, Alois; Herlth, Klaus P. (Hrsg.): Lebenslauf und Familienentwicklung. Mikroanalysen des Wandels familialer Lebensformen. Opladen: Leske \& Budrich: 7-37.

Meulemann, Heiner 1995: Die Geschichte einer Jugend. Lebenserfolg und Erfolgsdeutung ehemaliger Gymnasiasten zwischen dem 15. und 30. Lebensjahr. Opladen: Westdeutscher Verlag.

Mitchell, Barbara A. 1994: Family Structure and Leaving the Nest: A Social Resource Perspective. In: Sociological Perspectives 37,4: 651-671.

Nave-Herz, Rosemarie 2002: Wandel und Kontinuität in der Bedeutung, in der Struktur und Stabilität von Ehe und Familie in Deutschland. In: Nave-Herz, Rosemarie (Hrsg.): Kontinuität und Wandel der Familie in Deutschland. Eine zeitgeschichtliche Analyse. Stuttgart: Lucius und Lucius: 45-70.

Papastefanou, Christiane 1997: Auszug aus dem Elternhaus: Aufbruch und Ablösung im Erleben von Eltern und Kindern. Weinheim: Juventa Verlag.

Papastefanou, Christiane 2000: Der Auszug aus dem Elternhaus - ein vernachlässigter Gegenstand der Entwicklungspsychologie. In: Zeitschrift für Soziologie der Erziehung und Sozialisation 20,1: 55-69.

Pinquart, Martin; Silbereisen, Rainer K. 2007: Familienentwicklung. In: Brandstädter, Jochen; Lindenberger, Ulman (Hrsg.): Entwicklungspsychologie der Lebensspanne. Ein Lehrbuch. Stuttgart: Kohlhammer: 483-509.

Radloff, Lenore Sawyer 1980: Depression and the Empty Nest. In: Sex Roles 6,6: 775-781.

Scherger, Simone 2007: Destandardisierung, Differenzierung, Individualisierung. Westdeutsche Lebensläufe im Wandel. 1. Aufl. Wiesbaden: VS Verlag für Sozialwissenschaften.

Scherger, Simone 2009: Social change and the timing of family transitions in west germany. Evidence from cohort comparison. In: Time and Society 18,1: 106-129 [doi: 10.1177/0961463X08099947].

Szydlik, Marc; Schupp, Jürgen 1998: Stabilität und Wandel von Generationenbeziehungen. In: Zeitschrift für Soziologie 27,4: 297-315.

Wagner, Michae/ 1989: Räumliche Mobilität im Lebensverlauf: eine empirische Untersuchung sozialer Bedingungen der Migration. Stuttgart: Enke Verlag.

Wawrzyniak, Barbara 2014: Die subjektive Bedeutung der Familie in der nachelterlichen Phase. Phil. Diss. Essen-Duisburg.

Weick, Stefan 1993: Determinanten des Auszugs aus der elterlichen Wohnung. In: Dieckmann, Andreas; Weick, Stefan (Hrsg.): Der Familienzyklus als sozialer Prozeß - Bevölkerungssoziologische Untersuchung mit den Methoden der Ereignisanalyse. Berlin: Duncker und Humblot: 86-108. 
Ziegler, Rolf; Schladt, Diana 1993: Auszug aus dem Elternhaus und Hausstandsgründung. In: Diekmann, Andreas; Weick, Stefan (Hrsg.): Der Familienzyklus als sozialer Prozess. Bevölkerungssoziologische Untersuchungen mit den Methoden der Ereignisanalyse. Berlin: Duncker \& Humblot.

Eine Übersetzung dieses begutachteten und von der Autorin autorisierten deutschen Originaltextes durch das Bundesinstitut für Bevölkerungsforschung ist unter dem Titel "Entry into the Postparental Phase of the Family Life Cycle", DOI 10.12765/CPoS-2015-01en bzw. URN urn:nbn:de:bibcpos-2015-01en2, auf http://wWw.comparativepopulationstudies.de verfügbar.

Dr. Barbara Wawrzyniak ( $₫)$. Universität zu Köln, Institut für Soziologie und Sozialpsychologie (ISS). Köln. Deutschland. E-Mail: barbara.wawrzyniak@gmx.net URL: http://www.iss-wiso.uni-koeln.de/wawrzyniak.html?\&L $=0$ 


\section{Comparative Population Studies}

WWW.comparativepopulationstudies.de

ISSN: 1869-8980 (Print) - 1869-8999 (Internet)

Published by / Herausgegeben von

Prof. Dr. Norbert F. Schneider

Federal Institute for Population Research

D-65180 Wiesbaden / Germany

\section{Managing Editor /}

Verantwortlicher Redakteur

Frank Swiaczny

\section{Assistant Managing Editor /}

Stellvertretende Redakteurin

Katrin Schiefer

\section{Copy Editor (German) /}

Lektorat (deutsch)

Dr. Evelyn Grünheid

\section{Layout / Satz}

Beatriz Feiler-Fuchs

E-mail:cpos@bib.bund.de

\section{Scientific Advisory Board /}

Wissenschaftlicher Beirat

Paul Gans (Mannheim)

Johannes Huinink (Bremen)

Michaela Kreyenfeld (Rostock)

Marc Luy (Wien)

Clara H. Mulder (Groningen)

Notburga Ott (Bochum)

Peter Preisendörfer (Mainz)

Zsolt Spéder (Budapest)
Board of Reviewers / Gutachterbeirat Martin Abraham (Erlangen)

Laura Bernardi (Lausanne)

Hansjörg Bucher (Bonn)

Claudia Diehl (Konstanz)

Andreas Diekmann (Zürich)

Gabriele Doblhammer-Reiter (Rostock) Jürgen Dorbritz (Wiesbaden)

Anette Eva Fasang (Berlin)

E.-Jürgen Flöthmann (Bielefeld)

Alexia Fürnkranz-Prskawetz (Wien)

Beat Fux (Salzburg)

Joshua Goldstein (Berkeley)

Karsten Hank (Köln)

Sonja Haug (Regensburg)

Hill Kulu (Liverpool)

Aart C. Liefbroer (Den Haag)

Kurt Lüscher (Konstanz)

Emma Lundholm (Umeå)

Nadja Milewski (Rostock)

Dimiter Philipov (Wien)

Roland Rau (Rostock)

Tomáš Sobotka (Wien)

Jeroen Spijker (Barcelona)

Olivier Thévenon (Paris)

Helga de Valk (Brussel)

Heike Trappe (Rostock)

Michael Wagner (Köln) 